\title{
Transcatheter arterial chemoembolization combined with Hippo/YAP inhibition significantly improve the survival of rats with transplanted hepatocellular carcinoma
}

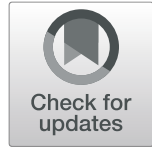

Yi Quan ${ }^{1 *}$, Zhi Li $^{2}$, Kangshun Zhu ${ }^{3}$ and Jundi Liang ${ }^{1}$

\begin{abstract}
Background: This study aimed to explore the effect of inhibiting the Hippo/Yes-associated protein (YAP) signaling pathway on the outcomes of transcatheter arterial chemoembolization (TACE) in treating transplanted hepatocellular carcinoma (HCC).

Methods: A transplanted HCC rat model was established. Then, rats were randomly divided into four groups: Sham, TACE, verteporfin (inhibitor of Hippo/YAP), and TACE+verteporfin. Lent-OE-YAP was transfected into rats to overexpress YAP in vivo. After treatments, morphological changes, tumor weight, and the overall survival of rats in different groups were analyzed. Real-time PCR, immunohistochemistry staining, and Western blotting were used to determine the expression of factors related to the Hippo/YAP signaling pathway.

Results: Tumor weight and tissue lesions in the TACE and verteporfin groups were significantly reduced compared with the Sham group. Verteporfin significantly decreased tumor weight after TACE treatment. In addition, verteporfin significantly improved the overall survival of rats with transplanted HCC after TACE treatment. Compared with the Sham group, both TACE and verteporfin groups exhibited significantly decreased expression of macrophage-stimulating (MST)1, MST2, long-acting thyroid stimulator 1, transcriptional co-activator with PDZbinding motif (TAZ), Yes-associated protein (YAP), TEA domain transcription factor (TEAD)1, TEAD2, TEAD3, and TEAD4. TACE plus verteporfin significantly enhanced the downregulation of effectors in the Hippo/YAP signaling pathway and decreased tumor size, while the overexpression of YAP exerted opposite effects.

Conclusion: The inhibition of the Hippo/YAP signaling pathway via verteporfin significantly improved the outcomes of TACE in treating transplanted HCC.

Keywords: Transplanted hepatocellular carcinoma, Transcatheter arterial chemoembolization, Hippo/YAP signaling pathway, Verteporfin, Prognosis
\end{abstract}

\footnotetext{
*Correspondence: quany_i@sina.com

'Department of Oncology Medilcal Center, The First People's Hospital of Zhaoqing, Zhaoqing, Guangdong 526000, China

Full list of author information is available at the end of the article
}

(C) The Author(s). 2021 Open Access This article is licensed under a Creative Commons Attribution 4.0 International License, which permits use, sharing, adaptation, distribution and reproduction in any medium or format, as long as you give appropriate credit to the original author(s) and the source, provide a link to the Creative Commons licence, and indicate if changes were made. The images or other third party material in this article are included in the article's Creative Commons licence, unless indicated otherwise in a credit line to the material. If material is not included in the article's Creative Commons licence and your intended use is not permitted by statutory regulation or exceeds the permitted use, you will need to obtain permission directly from the copyright holder. To view a copy of this licence, visit http://creativecommons.org/licenses/by/4.0/ The Creative Commons Public Domain Dedication waiver (http://creativecommons.org/publicdomain/zero/1.0/) applies to the data made available in this article, unless otherwise stated in a credit line to the data. 


\section{Introduction}

Hepatocellular carcinoma (HCC) is one of the most common malignancies worldwide and approximate $70 \%$ of the cases occur in Asian countries [1]. Many risk factors contribute to the development of HCC in humans, such as virus infection, chemicals, alcohol, and genetic factors [2, 3]. Hepatitis $\mathrm{B}$ and $\mathrm{C}$ virus ( $\mathrm{HBV}$ and $\mathrm{HCV}$ ) are two major risk factors for $\mathrm{HCC}[4,5]$. During $\mathrm{HBV}$ and $\mathrm{HCV}$ infection, environmental and genetic factors may promote the development of $\mathrm{HCC}[6,7]$. Despite multiple efforts that have been made, the pathological mechanisms of HCC remain unknown and need to be further elucidated.

The Hippo/Yes-associated protein (YAP) signaling pathway is commonly involved in the regulation of cell proliferation, differentiation, and metabolism $[8,9]$. The Hippo/YAP signaling pathway plays an essential role in HCC. The Hippo pathway is a suppressor that has been shown to inhibit cell growth when it is activated. However, gene fusion of its downstream effectors can lead to neoplasia by promoting cell proliferation, and decreasing apoptosis and differentiation [10]. YAP, as a pivotal downstream effector of the Hippo signaling pathway, functions mainly via the TEA domain (TEAD) family transcription factors to activate gene expression [11]. The transcriptional coactivator with PDZ binding motif (TAZ) is regulated by the Hippo pathway and is paralogous with YAP [12]. Zhang et al. showed that transforming growth factor beta 1 inhibited the proliferation of HCC tumor cells via targeting the Hippo signaling [13]. Fitamant et al. have found that YAP inhibition restored the differentiation of advanced $\mathrm{HCC}$ and thus regulated the development of tumor regression [14]. The Hippo/ YAP signaling also interacts with the Notch and Wnt/ $\beta$ catenin signaling pathways to repress the development of HCC [15]. In addition, the Hippo/YAP/TAZ signaling pathway regulates the pathogenesis of hepatic fibrosis to promote the formation of HCC [16]. However, the potential application of the Hippo/YAP signaling in clinics has not been reported.

Many efforts have been made to improve the prognosis of HCC and the main therapies include surgical resection, liver transplantation, chemotherapy, and locoregional treatment [17]. Transcatheter arterial chemoembolization (TACE) is a common treatment strategy for patients with unresectable HCC and often provides satisfying outcomes [18, 19]. Even though some retrospective studies have suggested that TACE treatment after refractoriness may cause liver function deterioration [20], there is no consensus on the refractoriness and unsuitability of TACE on treating HCC. Some studies have elicited that the effects of combined sorafenib with TACE for patients with HCC were uncertain [21, 22 ]. Hence, it is important to improve the application of
TACE in treating HCC. In 2017, a research team reported that the inhibition of the Wnt/ $\beta$-catenin signaling pathway significantly improved the therapeutic outcomes in TACE via attenuating the migration and invasion, and accelerating the apoptosis of tumor cells in rats with transplanted HCC [13]. Verteporfin, an FDAapproved drug blocking YAP-TEAD interaction and inhibiting YAP transcription [23], is commonly used as a pharmacological inhibitor. Verteporfin in combination with chemotherapeutic agents could overcome side effects caused by anti-cancer drugs in the treatment of HCC [24]. Zhao et al. found that evodiamine inhibited cell proliferation and facilitate apoptosis via the Hippo/ YAP signaling pathway in HCC [25]. Chai et al. reported that cucurbitacin $\mathrm{B}$ inhibited the expression of proteins related to the Hippo/YAP signaling pathway to inhibit proliferation and induce apoptosis [26]. However, the effect of inhibiting the Hippo/YAP signaling pathway in TACE-treated transplanted HCC has not been reported.

This study aimed to explore the effect of the Hippo/ YAP signaling pathway on the prognosis of TACE in treating transplanted HCC. It provided new information for improving the outcomes of TACE and using verteporfin to treat transplanted $\mathrm{HCC}$.

\section{Materials and methods}

\section{Construction of transplanted HCC model}

This study was approved by the animal ethical standard. Forty-two Sprague-Dawlay (SD) rats, weighing $90 \pm 10 \mathrm{~g}$ ( 5 weeks old), were used in this study (Shanghai Institute of Pharmaceutical Industry, Shanghai, China). Rats were kept under a specific pathogen free (SPF) condition (24$26^{\circ} \mathrm{C}, 45-55 \%$ humidity) with free access to water. Walker-256 tumors (Shanghai Institute of Pharmaceutical Industry, Shanghai, China) were used to conduct the HCC model. Subsequently, two rats were intraperitoneally injecting with $5 \times 10^{5}$ cells. One week later, rats were sacrificed. Ascites tumor, after formed, was extracted to obtain $800 \mathrm{~g}$ of ascites. Then ascites was centrifuged and washed with physiological saline. After that, the mushy cytoplasm was collected for further use. One hundred male SD rats weighed approximately $225 \pm 25$ g was purchased from SIPI (Shanghai, China) and a transplanted HCC model was constructed as previously reported [27]. Ten days later, the tumor of liver lobe $(1 \mathrm{~cm}$ in diameter) in rat was observed, indicating successful establishment of the model.

\section{Treatment for transplanted HCC model}

A total of 100 transplanted HCC model rats were randomly divided into four groups: the sham group $(n=25)$, the TACE group $(n=25)$, the verteporfin group $(n=25)$, and the TACE+verteporfin group $(n=25)$. Rats in the sham group underwent laparotomy without any other 
surgery. Rats in the TACE and TACE+verteporfin groups received gradual incision of the abdominal cavity by TACE. Rats in the verteporfin group were treated with $10 \mathrm{mg} / \mathrm{kg}$ verteporfin (Selleck Chemicals, Houston, USA; verteporfin was dissolved in $20 \mu \mathrm{l}$ of dimethyl sulfoxide and diluted to $5 \%$ using saline to reduce its toxicity). Rats in the TACE+verteporfin group received verteporfin during TACE therapy. The hepatic artery and gastroduodenal artery of the model rats were removed during the whole course of treatment, and microcatheter was inserted through gastroduodenal artery. Then, $10 \mathrm{mg} / \mathrm{kg}$ verteporfin was injected into the rats for verteporfin treatment. All treatments lasted for 10 days. After that, in each group, 5 rats were sacrificed for pathological analysis and subsequent experiments and the rest rats were used to record the survival condition. To evaluate the effects of dimethyl sulfoxide, a vehicle group in which rats were treated with dimethyl sulfoxide diluted in 5\% saline was set. To investigate the molecular mechanism, vectors overexpressing YAP was constructed by HanBio Co. Ltd., Shanghai, China using the lentivirus expressing system. The lent-OE-YAP was transfected into rats via tail vein injection, while lent$\mathrm{OE}-\mathrm{NC}$ was used as the control.

\section{Hematoxylin \& Eosin (H\&E) staining}

After treatment, liver tumors of each group were harvested and weighed. Then, part of liver tumor tissues was fixed with $4 \%$ paraformaldehyde (PFA) (Thermo Fisher Scientific, Shanghai, China), dehydrated by gradient ethanol (Sigma, Shanghai, China) and embedded by paraffin. Subsequently, tissues were cut into $5-\mu \mathrm{m}$ section, dewaxed using xylene (Sigma, Shanghai, China), rehydrated with gradient ethanol (Sigma, Shanghai, China), and stained with hematoxylin and eosin (Nanjing Jiancheng, Nanjing, Jiangsu, China) according to manufacturer's protocols. Then, the pathological features of liver tumor tissues were analyzed under the light microscope.

\section{Immunohistochemistry (IHC)}

Paraffin-embedded slides of HCC tissue were heated at $60{ }^{\circ} \mathrm{C}$ for $1 \mathrm{~h}$. Slides hydrated with xylene (Sigma-Aldrich, St. Louis, MO, USA) and gradient alcohol (Sigma-Aldrich, St. Louis, MO, USA) were dewaxed. For antigen extraction, slides were put into diluted potassium citrate (Sigma, Shanghai, China) solution and microwave- heated at $90{ }^{\circ} \mathrm{C}$ for $10 \mathrm{~min}$. After cooling at room temperature, slides were rinsed with phosphate buffered saline (Mechanistic) (Sigma-Aldrich, St. Louis, MO, USA) for 3 times (5 min each time). Then, $3 \% \mathrm{H}_{2} \mathrm{O}_{2}$ (Sigma-Aldrich, St. Louis, $\mathrm{MO}$, USA) were added at room temperature. Subsequently, slides were blocked with $5 \%$ goat serum (SigmaAldrich, St. Louis, MO, USA) at room temperature for 20 $\sim 30 \mathrm{~min}$, followed by incubation with primary antibodies
(MST, MST2, LATS1, LATS2, YAP1, TAZ, TEAD1, TEAD2, TEDA3, TEAD4, GAPDH) at $4{ }^{\circ} \mathrm{C}$ overnight. All antibodies were rabbit resource, diluted at 1:200, and purchased from Abcam (Cambridge, MA, USA). After washed with PBS (Sigma-Aldrich, St. Louis, MO, USA) for three times, slices were incubated with a goat-anti rabbit secondary antibody (Thermo Fisher Scientific, Shanghai, China) at room temperature for $1 \mathrm{~h}$ and then visualized using the DAB method (ZSGB-BIO, Beijing, China) according to manufacturer's protocol. Subsequently, slices were stained with hematoxylin (Nanjing Jiancheng, Nanjing, Jiangsu, China) according to manufacturer's protocols to visualize nuclei. For each sample, three fields of view were selected at random. Positive cells were counted.

\section{Quantitative real time PCR (RT-qPCR)}

After treatment, TRIzol reagent (Takara, Dalian, Shenyang, China) was utilized to extract total RNA according to manufacturer's protocol and a cDNA synthesis kit (Tiangen, Beijing, China) was utilized to synthesize cDNA according to manufacturer's instruction. The expressions of MST, MST2, LATS1, LATS2, YAP1, TAZ, TEAD1, TEAD2, TEDA3, TEAD4, and GAPDH (Abcam, Cambridge, MA, USA) were determined using SYBR Green Master Mix (Vazyme Biotech Co., Ltd., Nanjing, China)

Table 1 The primer sequences for RT-qPCR

\begin{tabular}{|c|c|}
\hline Gene type & Primer sequence \\
\hline \multirow[t]{2}{*}{ MST } & Forward: 5'-CCTCCCACATTCCGAAAACCA-3' \\
\hline & Reverse: 5'-GCACTCCTGACAAATGGGTG-3' \\
\hline \multirow[t]{2}{*}{ MST2 } & Forward: 5'-AGGAACA-GCAACGAGAATTGG-3' \\
\hline & Reverse: 5'-CCCCTTCACTCATCGTGCTT-3' \\
\hline \multirow[t]{2}{*}{ LATS1 } & Forward: 5'-AATTTGGGACGCATCATAAAGCC-3' \\
\hline & Reverse: 5'-TCGTCGAGGATCTTGGTAACTC-3' \\
\hline \multirow[t]{2}{*}{ LATS2 } & Forward: 5'-GCTTCATCCACCGAGACATCAA-3' \\
\hline & Reverse: 5'-CGACAGTTAGACACATCATCCCAGA-3' \\
\hline \multirow[t]{2}{*}{ YAP1 } & Forward: 5'-CCTGATGGATGGGAACAAGC-3' \\
\hline & Reverse:: 5'-GCACTCTGACTGATTCTCTGG-3' \\
\hline \multirow[t]{2}{*}{ TAZ } & Forward: 5'-ACGTCCTTCCTAACAGTCC-3' \\
\hline & Reverse: 5'-TGCCTGACTCTTCAGATGC-3' \\
\hline \multirow[t]{2}{*}{ TEAD1 } & Forward: 5'-CGCCTTCTTCCTCGTCAA-3' \\
\hline & Reverse: 5'-TCGCATACTCCGTCTCTAC-3' \\
\hline \multirow[t]{2}{*}{ TEAD2 } & Forward: 5'-CCACATGCCTTCTTCCTCGTCAA-3' \\
\hline & Reverse: 5'-CCGTCTCCACCTTCTCTACCACTT-3' \\
\hline \multirow[t]{2}{*}{ TEAD3 } & Forward: 5'-TCGGCAAGCAGGTGGTAGAGAAG-3' \\
\hline & Reverse: 5'-CAGGCAGGTGTGTGGAGGATGT-3' \\
\hline \multirow[t]{2}{*}{ TEAD4 } & Forward: 5'-CTGACGGAGGAAGGCAAGATGTATG-3' \\
\hline & Reverse: 5'-ACGGGCAAGCACCTGGATGT-3' \\
\hline \multirow[t]{2}{*}{ GAPDH } & Forward: 5'-CCTTCCGTGTTCCTACCCC-3' \\
\hline & Reverse: 5'-GCCCAAGATGCCCTTCAGT-3' \\
\hline
\end{tabular}


on a BioRad CFX96 Sequence Detection System (BioRad company, Berkeley. CA, USA). The primers (synthesized by Bo shang Biotechnology Co., Ltd., Shanghai, China) used for reaction are shown in Table 1. GAPDH was used as the internal control. The $2^{-\Delta \Delta C t}$ method was used to detect the relative expression of linc01014. The premier sequences were purchased from RiBio (Guangzhou, China).

\section{Western blotting}

A total of $0.05 \mu \mathrm{g}$ tissue samples were collected using RIPA lysis buffer (Millipore, Billerica, MA, USA) containing protease inhibitors (Sigma, Shanghai, China). Protein samples were centrifuged and collected. The BCA method (Pierce, Rockford, IL, USA) was used to determine the concentration according to manufacturer's protocol (Beyotime, Nanjing, Jiangsu, China). Subsequently, proteins as well as loading buffer were heated, after which proteins were separated by $10 \%$ SDS electrophoresis and transferred to polyvinylidene fluoride membranes (GVS Technology Co., Ltd., Suzhou, China). After blocked with milk at room temperature for one hour, membranes were incubated in a 1:1000 solution of primary antibodies
(MST, MST2, LATS1, LATS2, YAP1, TAZ, TEAD1, TEAD2, TEDA3, TEAD4, GAPDH; Abcam, Cambridge, MA, USA) at $4{ }^{\circ} \mathrm{C}$ overnight. Subsequently, membranes were incubated with the secondary antibody at room temperature for one hour and observed using the ECL method (Pierce, Rockford, IL, USA).

\section{Statistical analyses}

SPSS 19.0 (SPSS Incorporation, Chicago, IL, USA) was used for statistical analysis. Data were presented as mean \pm standard deviation (SD). Comparisons among groups were analyzed by student's t-test or one-way analysis. $P<0.05$ was considered statistically significant.

\section{Results \\ Verteporfin improves the effect of TACE in alleviating transplanted HCC}

After the transplanted HCC model was constructed, tumor weight of transplanted HCC was measured. The tumor weights and tumor weight/body weight ratio in the TACE, verteporfin, and TACE+verteporfin groups were markedly reduced compared with the Sham group

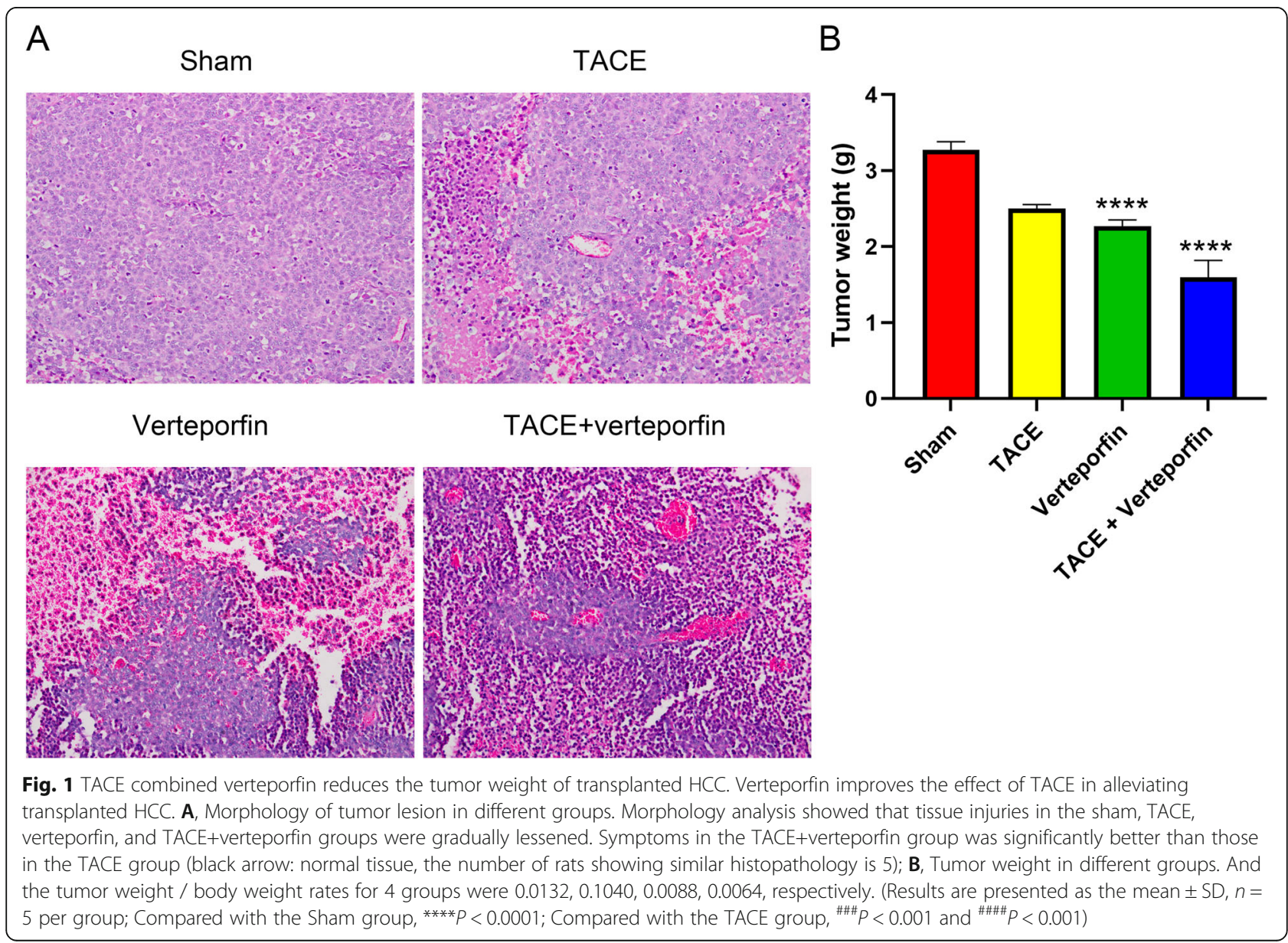




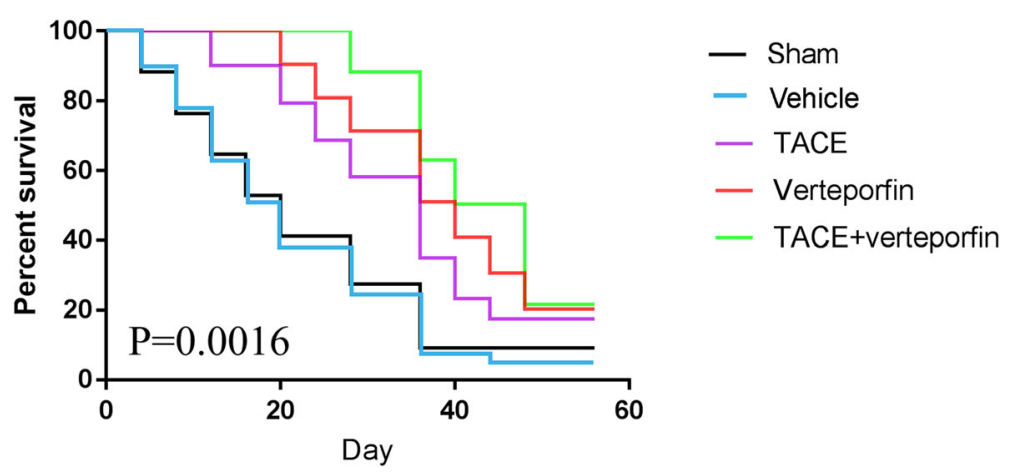

Fig. 2 Overall survival analysis for transplanted HCC rats in different groups. Verteporfin improves survival of rats after TACE therapy. The overall survival (OS) of rats in different groups were analyzed by Kaplan-Meier curve. (Results are presented as the mean \pm SD, $n=20$ per group)

$(P<0.05)$ (Fig. 1B). Meanwhile, the tumor weight of the TACE+verteporfin group was significantly lower than that of the TACE group $(P<0.05)$. The results of the vehicle group revealed that dimethyl sulfoxide used in the present study had no significant effect on tumor or body weight. Morphological analysis showed that tissue injuries in the
TACE, verteporfin, and TACE+verteporfin groups were alleviated compared with the Sham group and the symptoms in the TACE+verteporfin group was significantly better than those in the TACE group (Fig. 1A). These results showed that TACE combined verteporfin significantly suppressed the development of transplanted HCC.

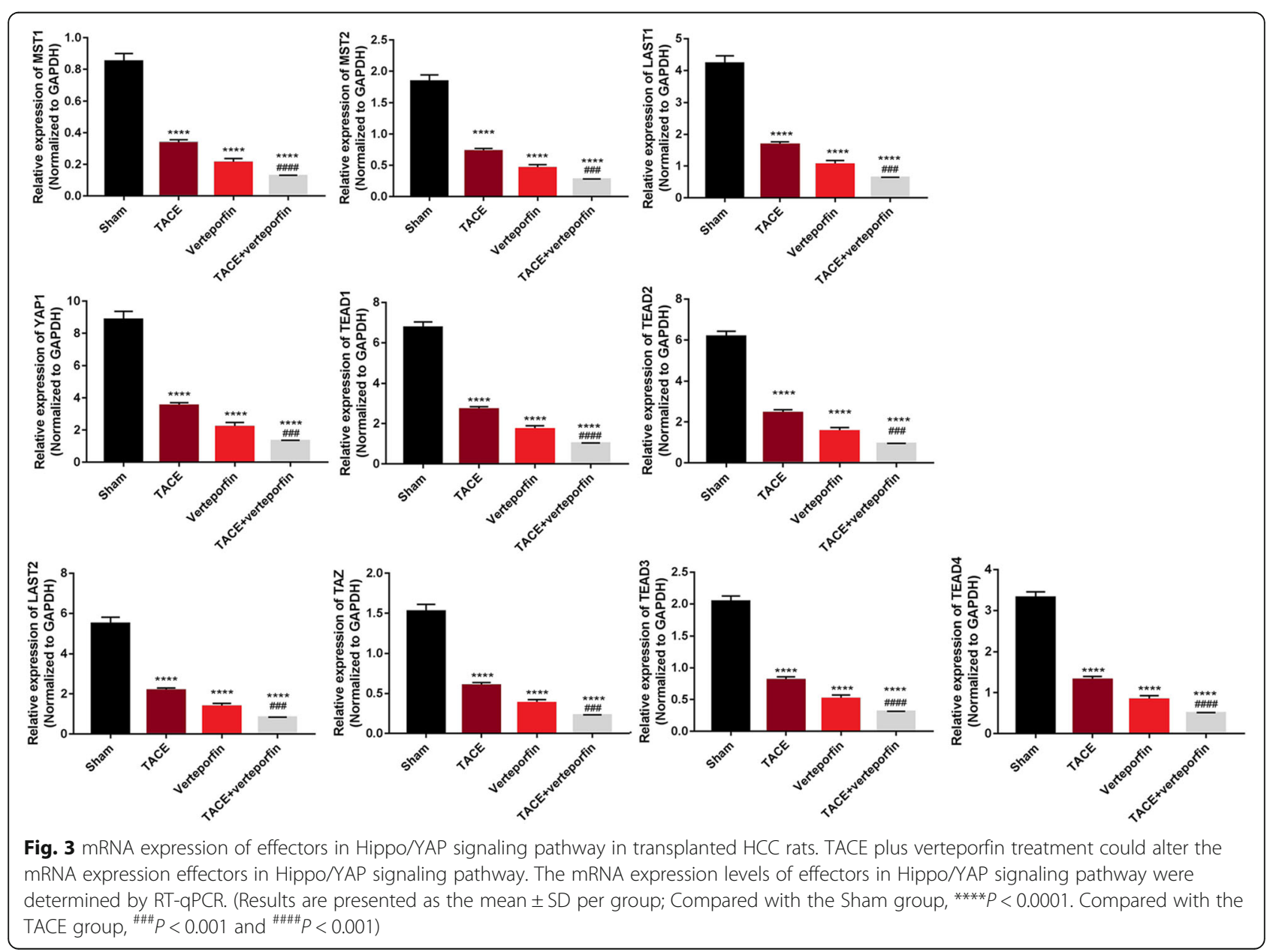


Verteporfin improves survival of rats after TACE therapy To further investigate the efficacy of TACE+verteporfin in treating transplanted $\mathrm{HCC}$, the overall survival (OS) of rats in different groups was analyzed using KaplanMeier curve (survival endpoint was measured on the death of rats). The median survival time of the Sham, TACE, verteporfin, and TACE+verteporfin groups were 20d, 36d, 40d, and 48d, respectively. The OS rate of the Sham group was dramatically lower than that in the TACE, verteporfin, and TACE+verteporfin groups $(P<$ $0.05)$. The TACE+verteporfin group showed significantly higher OS rate than that of the TACE group $(P<0.05)$ (Fig. 2). No significant difference was observed in the survival rate between the vehicle and Sham groups. These findings suggested that inhibiting the YAP signaling significantly improved the prognosis of $\mathrm{HCC}$ after TACE treatment.

TACE plus verteporfin treatment could alter the mRNA expression effectors in hippo/YAP signaling pathway To confirm the involvement of the Hippo/YAP signaling pathway, the mRNA expression of the effectors in the
Hippo/YAP signaling pathway was determined by RTqPCR. Both TACE and verteporfin significantly decreased the mRNA expressions of MST1, MST2, LAST1, LAST2, TAZ, YAP, TEAD1, TEAD2, TEAD3, and TEAD4 compared with the Sham group $(P<0.001)$. Moreover, TACE plus verteporfin significantly enhanced the downregulation of MST1, MST2, LAST1, LAST2, TAZ, YAP, TEAD1, TEAD2, TEAD3, and TEAD4 compared with the TACE group (Fig. $3, P<0.001$ ). These findings suggested that TACE plus verteporfin significantly inhibited the YAP signaling in transplanted HCC.

TACE plus verteporfin treatment could alter the protein expression effectors in hippo/YAP signaling pathway IHC was also performed to determine the expression of effectors in the Hippo/YAP signaling pathway. The result demonstrated that TACE and verteporfin significantly decreased the protein expressions of MST1, MST2, LAST1, TAZ, YAP, TEAD1, TEAD2, TEAD3, and TEAD4. TACE plus verteporfin significantly enhanced the expression of these proteins (Fig. 4).

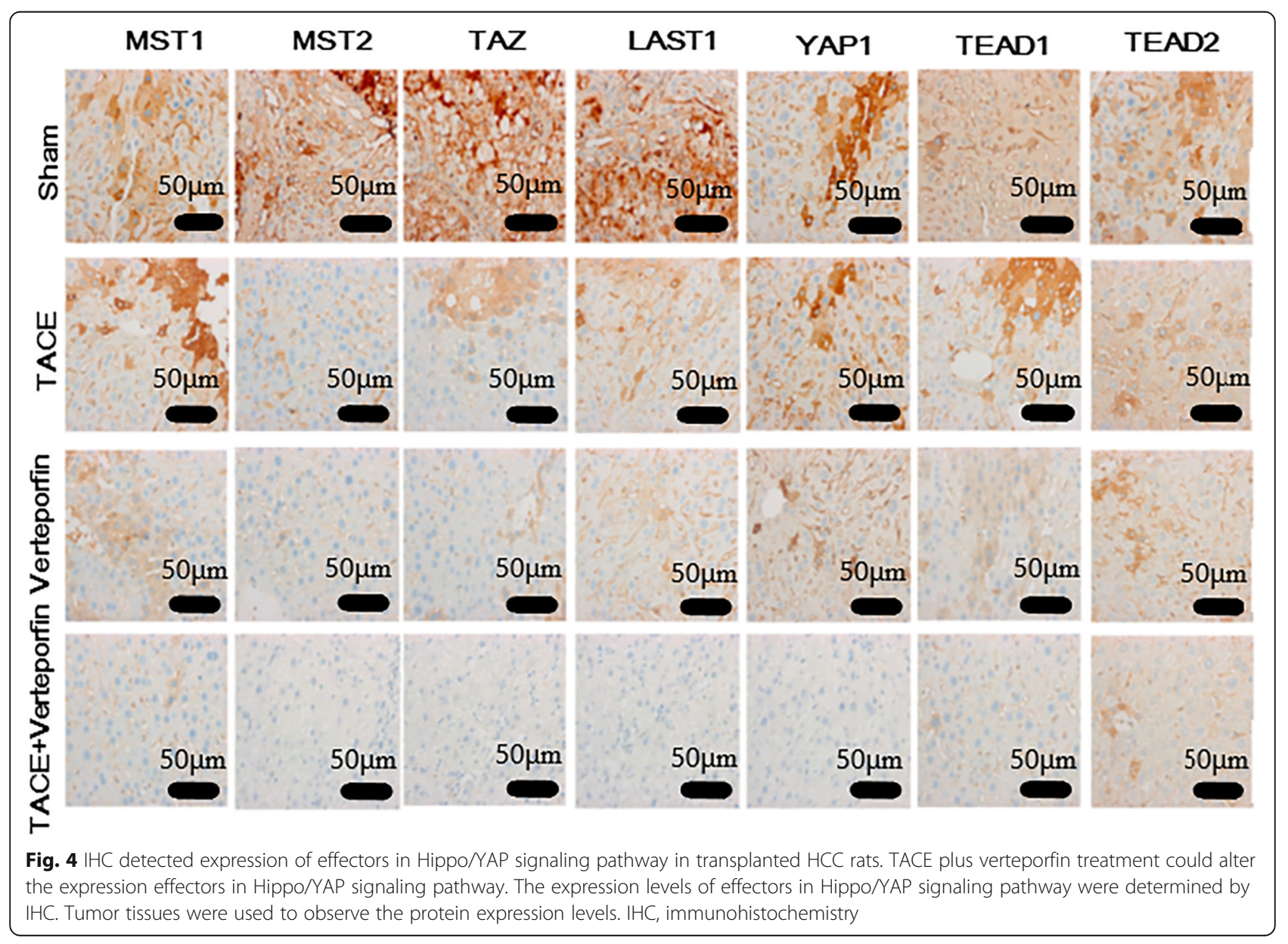


Overexpression of hippo/YAP signaling pathway reversed the effects of TACE plus verteporfin treatment

Furthermore, the protein levels of effectors in the Hippo/YAP signaling pathway were detected by Western blotting and IHC. Western blotting analysis suggested that both TACE and verteporfin significantly decreased the expressions of MST1, MST2, LAST1, TAZ, YAP, TEAD1, and TEAD2, and TACE plus verteporfin significantly enhanced these reductions (Fig. 5). However, the overexpression of YAP reversed the downregulation of these proteins compared to the OE-NC group. As shown in Fig. 6, the tumor size was decreased after treatment with TACE or verteporfin or both, while the overexpression of YAP resulted in the increase in the tumor size similar to the Sham group. The vehicle group showed that dimethyl sulfoxide had no significant effect on neither gene expression nor tumor growth. These data suggested that the overexpression of the Hippo/YAP signaling pathway reversed the effects of TACE plus verteporfin treatment.

\section{Discussion}

$\mathrm{HCC}$ is one of the most common cancers and the third leading cause of tumor-related mortality worldwide. $\mathrm{HCC}$-associated death is increased significantly in recent years [28]. Despite the efforts that have been made in recent decades, the outcomes and prognosis of patients with HCC remain dismal. TACE is a common therapeutic method for patients with intermediate-stage HCC and is of vital importance in alleviate the pathological condition of HCC patients [29]. However, there are still no global accepted guidelines for TACE treatment and the clinical outcomes vary among different individuals [30]. A study has demonstrated that sorafenib combined TACE treatment significantly improved the prognosis of patients with HCC [31], providing us a new insight in

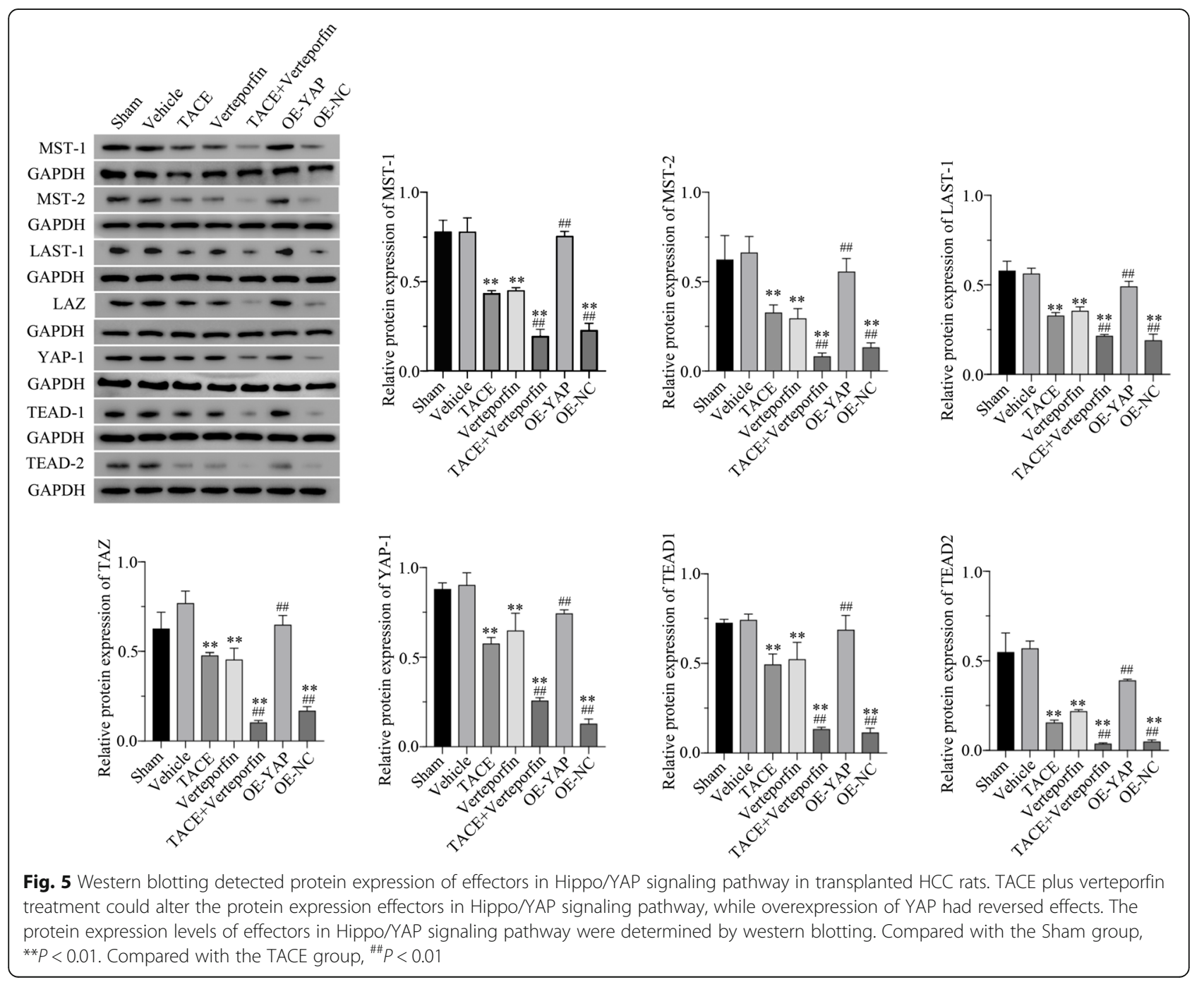




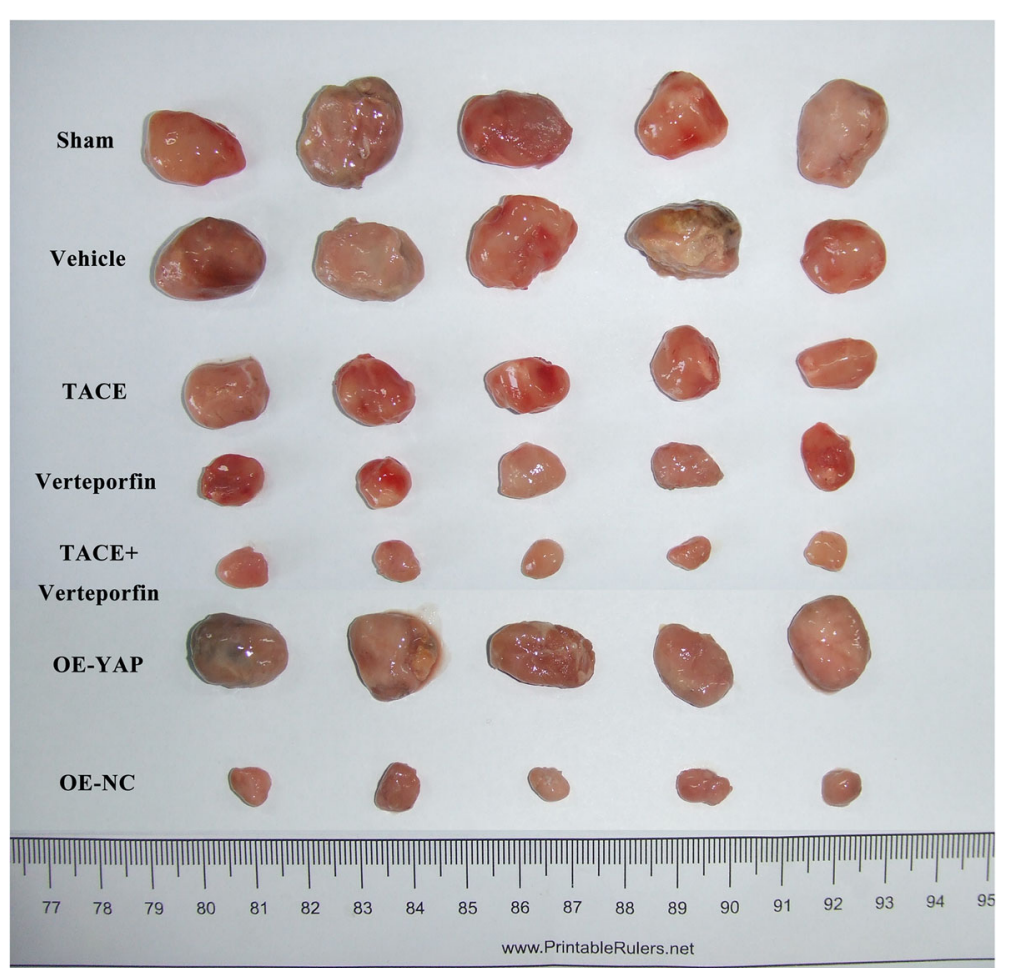

Fig. 6 The tumor size of transplanted HCC. The tumor size was detected in the sham, vehicle, TACE, verteporfin, TACE+verteporfin, OE-YAP and OE-NC groups. TACE plus verteporfin treatment could decrease the tumor size, while overexpression of YAP increased tumor size

treating HCC. However, Lencioni et al. revealed that sorafenib combined TACE with doxorubicin-eluting beads did not improve the time-to-tumor progression compared with TACE with doxorubicin-eluting beads in clinics [32]. Thus, further investigation is needed to confirm these findings.

Previous studies have reported that the Hippo/YAP signaling pathway plays a crucial role in the development of HCC [33]. Verteporfin has been reported to be an inhibitor of the Hippo/YAP signaling pathway. In this study, the effect of inhibiting the Hippo/YAP signaling pathway on the outcomes of TACE in treating transplanted HCC was explored. The results showed that both TACE and verteporfin treatment reduced tumor weight and lesion and improved the OS ratio of transplanted HCC, indicating that the Hippo/ YAP signaling pathway might have a significant impact on the pathogenesis of transplanted HCC. Interestingly, TACE combined verteporfin significantly improved the outcomes and prognosis of transplanted HCC compared with the TACE group, suggested that inhibiting the Hippo/YAP signaling pathway could significantly improve the outcomes of TACE in treating transplanted HCC. Previous study has revealed that YAP led to chromosomal instability in liver cancer [34], which might explain that inhibiting the
Hippo/YAP signaling pathway improved the outcomes of TACE in treating transplanted HCC.

The effectors of the Hippo/YAP signaling pathway were detected in each group. Both TACE and verteporfin significantly decreased the expressions of MST1, MST2, LAST1, LAST2, TAZ, YAP, TEAD1, TEAD2, TEAD3, and TEAD4 $[35,36]$ compared with the Sham group. TACE plus verteporfin significantly enhanced these reductions. Thus, a combination of verteporfin and TACE suppressed the Hippo/YAP pathway by downregulating the expressions of these effectors. Previous study showed that LAST2 mediated the phosphorylation of YAP to regulate the pathogenesis of HCC [37]. Zhang et al. revealed that YAP interacted with HIF-1a to promote the stability of HIF-1a and induced cell glycolysis under hypoxic stress, indicating that inhibiting YAP expression could suppress the glycolysis of tumor, the energy provider for $\mathrm{HCC}$ [38]. Integrin $\alpha 2 \beta 1$ inhibits the phosphorylation of MST1 and activates the YAP signaling to promote the pathogenesis of HCC [39]. A recent study has also revealed that YAP/TAZ could be used as an diagnostic indicator for HCC [40]. In addition, NUP37 positively regulated the YAP/TEAD signaling to promote the development of HCC [41]. These findings suggested that TACE combined verteporfin significantly suppressed the development of transplanted HCC by 
inhibiting the expression of effectors in the Hippo/YAP signaling pathway.

\section{Study strength and limitations}

The novel aspect of the current study was that the effect of inhibiting the Hippo/YAP signaling pathway in TACE-treated transplanted HCC was explored in vivo. The results highlighted the critical role of the Hippo/ YAP signaling in the prognosis of TACE-treated transplanted HCC. The limitations of this study should also be addressed. First, the potential mechanism by which TACE plus verteporfin influences the Hippo/YAP pathway remains to be investigated. Second, other pathways independent of Hippo/YAP-associated factors were not taken into account; therefore, whether verteporfin affects other pathways is still unclear. Third, the effect of TACE combined with verteporfin on other organs is unknown.

\section{Conclusion}

This study shows that the Hippo/YAP signaling pathway plays a critical role in the development of transplanted HCC. The inhibition of the Hippo/YAP signaling pathway by its inhibitor significantly improved the outcomes of TACE in treating transplanted HCC. These results suggest that TACE combined with verteporfin is a potential therapeutic method for HCC treatment.

\section{Supplementary Information}

The online version contains supplementary material available at https://doi. org/10.1186/s12944-021-01486-w.

Additional file 1: Figure S1. Validation of the specificity of the antibody in IHC. The expression levels of effectors in Hippo/YAP signaling pathway in normal liver tissues vs liver tumor tissues were determined by $\mathrm{IHC}$.

\section{Acknowledgements}

We would like to acknowledge the everyone for their helpful contributions on this paper.

\section{Authors' contributions}

Each author has made an important scientific contribution to the study and has assisted with the drafting or revising of the manuscript. The authors read and approved the final manuscript.

\section{Funding}

Project of Guangdong Science and Technology Department (No:2011B031800294)

Availability of data and materials

The data are free access to available upon request.

\section{Declarations}

\section{Ethics approval and consent to participate}

Identifying the Second Affiliated Hospital of Guangzhou Medical University and licensing committee approving the experiments, including any relevant details; Confirming that all experiments were performed in accordance with relevant guidelines and regulations. All animal experiments were approved by the Animal Research Ethics Committee of Second Affiliated Hospital of Guangzhou Medical University, Guangzhou, China.
Consent for publication

All of the authors have consented to publish this research.

\section{Competing interests}

All authors declare no conflict of interest.

\section{Author details}

${ }^{1}$ Department of Oncology Medilcal Center, The First People's Hospital of Zhaoqing, Zhaoqing, Guangdong 526000, China. ${ }^{2}$ Department of Interventional, First Affiliated Hospital of Suzhou University, Suzhou, Jiangsu 215006, China. ${ }^{3}$ Department of Minimally Invasive Medicine, Second Affiliated Hospital of Guangzhou Medical University, Guangzhou 510000, China.

Received: 18 June 2020 Accepted: 28 May 2021

Published online: 25 July 2021

\section{References}

1. Chow PKH, Li L, Li J, Jia F, Wang H-J, Hasegawa K, et al. Differences in presentation and management patterns in patients with hepatocellular carcinoma (HCC): data from HCC registry in Asia. J Clin Oncol. 2019;37(4_ suppl):229. https://doi.org/10.1200/JCO.2019.37.4_suppl.229.

2. Bruix J, Reig M, Sherman M. Evidence-based diagnosis, staging, and treatment of patients with hepatocellular carcinoma. Gastroenterology. 2016;150(4):835-53. https://doi.org/10.1053/j.gastro.2015.12.041.

3. Sparkman J, Hernaez R, El-Serag HB. Traditional Chinese medicine to the rescue of allopathic medicine in the co-adjuvant treatment of hepatocellular carcinoma. Transl Gastroenterol Hepatol. 2018;3:97. https:// doi.org/10.21037/tgh.2018.11.05.

4. Maucort-Boulch D, de Martel C, Franceschi S, Plummer M. Fraction and incidence of liver cancer attributable to hepatitis $B$ and $C$ viruses worldwide. Int J Cancer. 2018;142(12):2471-7. https://doi.org/10.1002/ijc.31280.

5. Petruzziello A. Epidemiology of hepatitis B virus (HBV) and hepatitis $C$ virus (HCV) related hepatocellular carcinoma. Open Virol J. 2018;12(1):26-32. https://doi.org/10.2174/1874357901812010026.

6. An P, Xu J, Yu Y, Winkler CA. Host and viral genetic variation in HBV-related hepatocellular carcinoma. Front Genet. 2018:9:261. https://doi.org/10.3389/ fgene.2018.00261.

7. Axley P, Ahmed Z, Ravi S, Singal AK. Hepatitis C virus and hepatocellular carcinoma: a narrative review. J Clin Transl Hepatol. 2018;6(1):79-84. https:// doi.org/10.14218/JCTH.2017.00067.

8. Ma Y, Yang Y, Wang F, Wei Q, Qin H. Hippo-YAP signaling pathway: a new paradigm for cancer therapy. Int J Cancer. 2015;137(10):2275-86. https://doi. org/10.1002/ijc.29073.

9. Yimlamai D, Fowl BH, Camargo FD. Emerging evidence on the role of the hippo/YAP pathway in liver physiology and cancer. J Hepatol. 2015;63(6): 1491-501. https://doi.org/10.1016/j.jhep.2015.07.008.

10. Plouffe SW, Hong AW, Guan KL. Disease implications of the hippo/YAP pathway. Trends Mol Med. 2015;21(4):212-22. https://doi.org/10.1016/j. molmed.2015.01.003.

11. Yan B, Li T, Shen L, Zhou Z, Liu X, Wang X, et al. Simultaneous knockdown of YAP and TAZ increases apoptosis of hepatocellular carcinoma cells under hypoxic condition. Biochem Biophys Res Commun. 2019;515(2):275-81. https://doi.org/10.1016/j.bbrc.2019.05.143.

12. Lei QY, Zhang $H$, Zhao B, Zha ZY, Bai F, Pei $X H$, et al. TAZ promotes cell proliferation and epithelial-mesenchymal transition and is inhibited by the hippo pathway. Mol Cell Biol. 2008;28(7):2426-36. https://doi.org/10.1128/ MCB.01874-07.

13. Zhang $X$, Fan Q, Li Y, Yang Z, Yang L, Zong Z, et al. Transforming growth factor-beta1 suppresses hepatocellular carcinoma proliferation via activation of hippo signaling. Oncotarget. 2017;8(18):29785-94. https://doi.org/10.1 8632/oncotarget.14523.

14. Fitamant J, Kottakis F, Benhamouche S, Tian H, Chuvin N, Parachoniak C, et al. YAP inhibition restores hepatocyte differentiation in advanced HCC, Leading to Tumor Regression. Cell Reports. 2015;10(10):1692-707. https:// doi.org/10.1016/j.celrep.2015.02.027.

15. Kim W, Khan SK, Gvozdenovic-Jeremic J, Kim Y, Dahlman J, Kim H, et al. Hippo signaling interactions with Wnt $/ \mathbb{I}^{2}$-catenin and notch signaling repress liver tumorigenesis. J Clin Investig. 2017;127:137-52.

16. Moon H, Cho K, Shin S, Kim DY, Han K-H, Ro SW. High risk of hepatocellular carcinoma development in fibrotic liver: role of the hippo-YAP/TAZ 
signaling pathway. Int J Mol Sci. 2019;20(3):581. https://doi.org/10.3390/ ijms20030581.

17. Watchmaker J, Cruz J, Albin M, Fritsche M, Su J, Fleming J, et al. 3:09 PM Abstract No. 34 Neutrophil to lymphocyte ratio (NLR) predicts early tumor progression in transarterial chemoembolization (TACE) for hepatocellular carcinoma (HCC): assessment by mRECIST. J Vasc Int Radiol. 2018:29:S19.

18. Ling Q, Liu J, Zhuo J, Zhuang R, Huang H, He X, et al. Development of models to predict early post-transplant recurrence of hepatocellular carcinoma that also integrate the quality and characteristics of the liver graft: a national registry study in China. Surgery. 2018;164(1):155-64. https:// doi.org/10.1016/j.surg.2018.01.022.

19. Lee SW, Lee HL, Han NI, Jang JW, Bae SH, Choi JY, et al. Early treatment response to transcatheter arterial chemoembolization is associated with time to the development of extrahepatic metastasis and overall survival in intermediate-stage hepatocellular carcinoma. Cancer Chemother Pharmacol. 2017;79(1):81-8. https://doi.org/10.1007/s00280-016-3202-8.

20. Kudo M, Raoul J-L, Lee HC, Cheng A-L, Nakajima K, Peck-Radosavljevic M. Deterioration of liver function after transarterial chemoembolization (TACE) in hepatocellular carcinoma (HCC): An exploratory analysis of OPTIMIS—An international observational study assessing the use of sorafenib after TACE. J Clin Oncol. 2018;36:368.

21. Zhou TY, Zhuang LH, Hu Y, Zhou YL, Lin WK, Wang DD, et al. Inactivation of hypoxia-induced YAP by statins overcomes hypoxic resistance tosorafenib in hepatocellular carcinoma cells. Sci Rep. 2016;6(1):30483. https://doi.org/1 $0.1038 /$ srep30483.

22. Liu L, Chen H, Wang M, Zhao Y, Cai G, Qi X, et al. Combination therapy of sorafenib and TACE for unresectable HCC: a systematic review and metaanalysis. PLoS One. 2014;9(3):e91124. https://doi.org/10.1371/journal.pone. 0091124.

23. Perra A, Kowalik MA, Ghiso E, Ledda-Columbano GM, Di Tommaso L, Angioni MM, et al. YAP activation is an early event and a potential therapeutic target in liver cancer development. J Hepatol. 2014;61(5):108896. https://doi.org/10.1016/j.jhep.2014.06.033.

24. Gavini J, Dommann N, Jakob MO, Keogh A, Bouchez LC, Karkampouna S, et al. Verteporfin-induced lysosomal compartment dysregulation potentiates the effect of sorafenib in hepatocellular carcinoma. Cell Death Dis. 2019; 10(10):749. https://doi.org/10.1038/s41419-019-1989-z.

25. Zhao S, Xu K, Jiang R, Li DY, Guo XX, Zhou P, et al. Evodiamine inhibits proliferation and promotes apoptosis of hepatocellular carcinoma cells via the hippo-yes-associated protein signaling pathway. Life Sci. 2020;251: 117424. https://doi.org/10.1016/j.Ifs.2020.117424.

26. Chai $Y$, Xiang $K$, Wu Y, Zhang T, Liu Y, Liu X, et al. Cucurbitacin B inhibits the hippo-YAP signaling pathway and exerts anticancer activity in colorectal Cancer cells. Med Sci Monit. 2018;24:9251-8. https://doi.org/10.12659/MSM. 911594.

27. Wang BM, Li N. Effect of the Wnt/ß-catenin signaling pathway on apoptosis, migration and invasion of transplanted hepatocellular carcinoma cells after transcatheter arterial chemoembolization in rats. J Cell Biochem. 2018; 119(5):4050-60. https://doi.org/10.1002/jcb.26576.

28. Bray F, Ferlay J, Soerjomataram I, Siegel RL, Torre LA, Jemal A. Global cancer statistics 2018: GLOBOCAN estimates of incidence and mortality worldwide for 36 cancers in 185 countries. CA Cancer J Clin. 2018;68(6):394-424. https://doi.org/10.3322/caac.21492.

29. Buckstein M, Kim E, Fischman A, Facciuto M, Sung M, Taouli B, et al. THU188 - phase II trial using combination of TACE and SBRT for unresectable single large HCC: preliminary report. J Hepatol. 2018;68:S211-S2. https://doi. org/10.1016/S0168-8278(18)30638-X.

30. Tsurusaki M, Murakami T. Surgical and Locoregional therapy of HCC: TACE. Liver Cancer. 2015;4(3):165-75. https://doi.org/10.1159/000367739.

31. Liu F, Meng Z, Shao G, Wang J, Wang Z, Yang J, et al. Patterns of sorafenib and TACE treatment of unresectable hepatocellular carcinoma in a Chinese population: subgroup analysis of the GIDEON study. Mol Biol Rep. 2017;44:1-10.

32. Lencioni R, Llovet JM, Han G, Tak WY, Yang J, Guglielmi A, et al. Sorafenib or placebo plus TACE with doxorubicin-eluting beads for intermediate stage HCC: the SPACE trial. J Hepatol. 2016;64(5):1090-8. https://doi.org/10.1016/j. jhep.2016.01.012.

33. Xia H, Dai X, Yu H, Zhou S, Fan Z, Wei G, et al. EGFR-PI3K-PDK1 pathway regulates YAP signaling in hepatocellular carcinoma: the mechanism and its implications in targeted therapy. Cell Death Dis. 2018;9(3):269. https://doi. org/10.1038/s41419-018-0302-x
34. Weiler S. YAP induces chromosomal instability in liver cancer patients. Pathologe. 2018;39(S2):185-8. https://doi.org/10.1007/s00292-018-0494-y.

35. Yuan H, Liu H, Liu Z, Zhu D, Amos Cl, Fang S, et al. Genetic variants in hippo pathway genes YAP1, TEAD1 and TEAD4 are associated with melanoma-specific survival. Int J Cancer. 2015;137(3):638-45. https://doi. org/10.1002/ijc.29429.

36. Mo JS, Park HW, Guan KL. The hippo signaling pathway in stem cell biology and cancer. EMBO Rep. 2014;15(6):642-56. https://doi.org/10.15252/embr.2 01438638.

37. Guo C, Wang X, Liang L. LATS2-mediated YAP1 phosphorylation is involved in HCC tumorigenesis. Int J Clin Exp Pathol. 2015;8(2):1690-7.

38. Zhang X, Li Y, Ma Y, Yang L, Wang T, Meng $X$, et al. Yes-associated protein (YAP) binds to HIF-1a and sustains HIF-1a protein stability to promote hepatocellular carcinoma cell glycolysis under hypoxic stress. J Exp Clin Cancer Res. 2018;37(1):216. https://doi.org/10.1186/s13046-018-0892-2.

39. Wong K-F, Liu AM, Hong W, Xu Z, Luk JM. Integrin a2 $\beta 1$ inhibits MST1 kinase phosphorylation and activates yes-associated protein oncogenic signaling in hepatocellular carcinoma. Oncotarget. 2016;7(47):77683-95. https://doi.org/10.18632/oncotarget.12760.

40. Van Haele M, Moya IM, Karaman R, Rens G, Snoeck J, Govaere O, et al. YAP and TAZ heterogeneity in primary liver Cancer: An analysis of its prognostic and diagnostic role. Int J Mol Sci. 2019;20(3):638. https://doi.org/10.3390/ ijms20030638.

41. Luo X, Liu Y, Feng W, Lei L, Du Y, Wu J, et al. NUP37, a positive regulator of YAP/TEAD signaling, promotes the progression of hepatocellular carcinoma. Oncotarget. 2017:8(58):98004-13. https://doi.org/10.18632/oncotarget.20336.

\section{Publisher's Note}

Springer Nature remains neutral with regard to jurisdictional claims in published maps and institutional affiliations.

Ready to submit your research? Choose BMC and benefit from:

- fast, convenient online submission

- thorough peer review by experienced researchers in your field

- rapid publication on acceptance

- support for research data, including large and complex data types

- gold Open Access which fosters wider collaboration and increased citations

- maximum visibility for your research: over $100 \mathrm{M}$ website views per year

At $\mathrm{BMC}$, research is always in progress.

Learn more biomedcentral.com/submissions 